\title{
Motion-Induced Transition of Positronium through a Static Periodic Magnetic Field in the Sub-THz Region
}

\author{
Y. Nagata $\odot,{ }^{1,}{ }^{*}$ K. Michishio $\odot,{ }^{2}$ T. Iizuka, ${ }^{1}$ H. Kikutani, ${ }^{1}$ L. Chiari $\odot{ }^{3}$ F. Tanaka, ${ }^{1}$ and Y. Nagashima $\odot^{1,4}$ \\ ${ }^{1}$ Department of Physics, Tokyo University of Science, 162-8601 Tokyo, Japan \\ ${ }^{2}$ National Institute of Advanced Industrial Science and Technology (AIST), \\ 305-8568 Ibaraki, Japan \\ ${ }^{3}$ Department of Applied Chemistry and Biotechnology, Chiba University, 263-8522 Chiba, Japan \\ ${ }^{4}$ Institute of Materials Structure Science, High Energy Accelerator Research Organization (KEK), \\ 1-1 Oho, Tsukuba, Ibaraki 305-0801, Japan
}

(Received 21 February 2020; accepted 7 April 2020; published 29 April 2020)

\begin{abstract}
Atoms moving in a static periodic field experience a time-dependent oscillating field in their own rest frame. By tuning the frequency, an atomic transition can be induced. So far, this type of transition has been demonstrated in the EUV region or at higher frequencies by crystalline fields and in the microwave region by artificial fields. Here, we present the observation of the transition of positronium (Ps) in the sub-THz region by using an energy-tunable Ps beam with a multilayered magnetic grating. This grating produces a microsized periodic field, whose amplitude corresponds to a huge energy flux of $\sim 100 \mathrm{MW} \mathrm{cm}^{-2}$, resulting in the efficient magnetic dipole transition.
\end{abstract}

DOI: 10.1103/PhysRevLett.124.173202

Atomic resonant transitions, usually induced by electromagnetic waves, such as lasers or microwaves, can also be induced by spatially static periodic fields. Atoms moving in such a field experience a time-dependent oscillating field in their own rest frame. When the oscillation frequency coincides with the atomic transition frequency, an atomic resonant transition, called "motion-induced transition" in this Letter, may occur.

The transition by a crystalline field was predicted by Okorokov theoretically in 1965 [1,2] and first demonstrated in 1978 for the $1 s-2 p$ transition in the EUV region of highly charged heavy ions [3], named "resonant coherent excitation" or "Okorokov effect." The crystalline field, whose strength was of the order of $100 \mathrm{GV} / \mathrm{m}$ [4], induces an efficient electric dipole transition. Manipulation of the energy levels of those ions was successfully accomplished in crystals [5-10] and at crystal surfaces [11-13]. Recently this method has been applied to the precision spectroscopy of Li-like $\mathrm{U}^{89+}$ irradiated on a Si crystal in the X-ray region to study QED effects at the $2 s$ energy level [14].

The transition with an artificial periodic structure was attempted, but only in the microwave region. The first transition by a static electric field was observed with a comb-shaped electrode for the $3 s-3 p$ Lamb shift of

Published by the American Physical Society under the terms of the Creative Commons Attribution 4.0 International license. Further distribution of this work must maintain attribution to the author(s) and the published article's title, journal citation, and DOI.
$520 \mathrm{MHz}$ of hydrogen already in 1969 [15]. However, the transition by a static magnetic field was first demonstrated much later, in 2005, with a periodically assembled current for the Zeeman sublevels of around $1 \mathrm{MHz}$ of $\mathrm{Rb}$ atoms [16-18] and then with a magnetic film $[19,20]$, i.e., so-called "motion-induced resonance." A neutron spin resonance of around $1 \mathrm{MHz}$ was also observed by using a periodic current sheet, i.e., a so-called "spatial spin resonance" [21,22].

The principle of this transition is simple. However, the feasibility of the transition depends on how the periodic field appropriate for the system is prepared. For example, the transition by a crystalline field was only performed for resonance frequencies higher than the EUV region, usually for highly charged heavy ions with injection energies higher than $\sim \mathrm{MeV} /$ nucleon to avoid collisions, which destroy the coherence. On the other hand, artificial periodic structures may enable us to overcome these limitations in the application of the transition, for example, through microfabrication [20].

This Letter presents the observation of the motioninduced transition in the sub-THz region for the positronium (Ps) atom, composed of an electron and a positron, by using a recently developed high-quality energy-tunable Ps beam [23] in combination with a multilayered magnetic grating. The microfabricated structure of the grating produces a microsized periodic magnetic field and keeps the coherence by ensuring that the Ps atoms pass through the slots.

A Ps atom has a hyperfine structure, which depends on the total spin $S=0$ and $S=1$, named para-positronium 


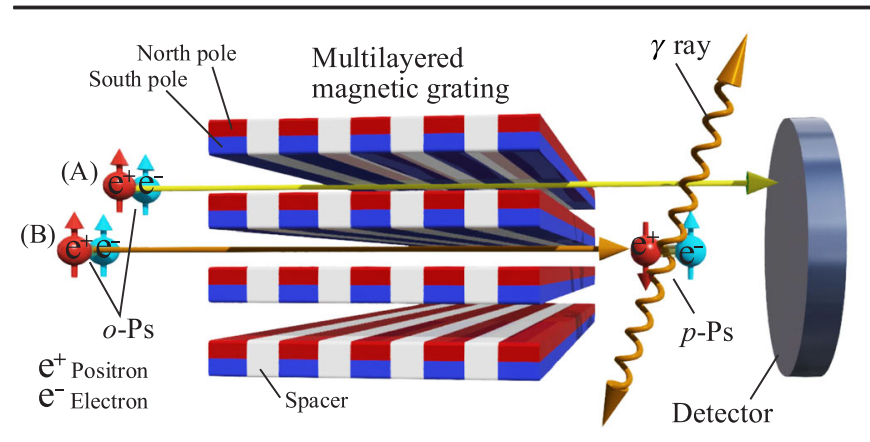

FIG. 1. Conceptual figure of our experiment. (A) Off-resonance case: $o$-Ps atoms pass through the multilayered magnetic grating and are counted by a detector. (B) On-resonance case: When $o$-Ps atoms pass through the grating, they transition to $p$-Ps atoms, which self-annihilate into two $\gamma$ rays almost immediately and, therefore, are not counted by the detector.

( $p$-Ps) and ortho-positronium (o-Ps), respectively. The lifetime of $p$-Ps is $125 \mathrm{ps}$, which is 3 orders of magnitude shorter than that of $o$-Ps (142 ns). The energy difference between them is $0.84 \mathrm{meV}(203 \mathrm{GHz})$ and was measured by a direct method [24,25] and indirect methods [26-28], using microwaves or lasers thus far. Although this transition is electric dipole forbidden, it can be induced by a magnetic dipole.

Figure 1 shows the concept of our experimental method. $o$-Ps atoms are directed through a magnetic grating, which has a periodic structure, generating a static periodic magnetic field of period length $a$. The atoms experience a time-dependent oscillating field with a frequency $f=\gamma v / a$, where $v$ and $\gamma$ are the velocity of the Ps atoms and the Lorentz factor, respectively. In the off-resonance condition (A), the $o$-Ps atoms exit the grating and then are counted by a detector. In the resonance condition (B), $o$-Ps transition to $p$-Ps, which decay into $2 \gamma$ rays by selfannihilation almost immediately and, hence, are not counted by the detector. Therefore, if we measure the number of $o$-Ps atoms as a function of $v$, the hyperfine resonance can be observed.

The condition required for this Ps hyperfine resonance is estimated by the Rabi oscillation [29]. When $v=1.8 \times 10^{7} \mathrm{~m} / \mathrm{s}$, corresponding to a kinetic energy of $1.8 \mathrm{keV}$, which is attainable with our Ps beam line, the resonance may occur for $a=90 \mu \mathrm{m}$. When passing through a path length corresponding to 10 periods, the Ps atom travels through the grating only for $50 \mathrm{ps}$. The Rabi oscillation shows that the magnetic field amplitude $B_{a}$ should be larger than $0.1 \mathrm{~T}$ to achieve an efficient transition. In the past experiments of this type of transition, $B_{a}$ was less than $1 \mathrm{mT}$.

To achieve this requirement, we used a ferromagnetic material made through microfabrication and designed a microsized structure, magnetized by permanent magnets. Figure 2(a) shows the whole setup of the multilayered magnetic grating. The central part is a multilayered

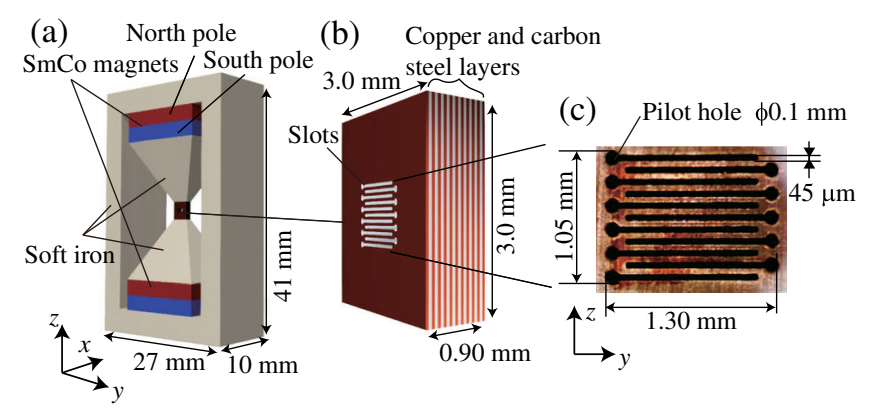

FIG. 2. Multilayered magnetic grating. (a) Whole setup of the multilayered magnetic grating. (b) Multilayered transmission grating, assembled by stacking 10 carbon steel foils $(50 \mu \mathrm{m}$ thickness) and 11 copper foils (40 $\mu$ m thickness) alternately and by using a diffusion bonding vacuum hot press. The grating was magnetized by two SmCo magnets $\left(\mathrm{Sm}_{2} \mathrm{Co}_{17}\right.$, grade 28$)$ that produced a static periodic magnetic field in the slots via two tapered soft iron blocks (SS400) to concentrate the magnetic flux on the transmission grating and achieve a higher field. (c) Photo of the 11 through slots $(45 \mu \mathrm{m} \times 1.30 \mathrm{~mm})$ made by wire electrical discharge machining for the transmission of the Ps atoms.

transmission grating, which consists of 10 layers of the periodic structure of carbon steel and copper foils, as shown in Fig. 2(b). The period length was estimated to be $86.4 \pm 0.4 \mu \mathrm{m}$ calculated from the thickness of the grating, where the uncertainty included the micrometer accuracy. For the transmission of the Ps atoms, 11 through slots $(45 \mu \mathrm{m} \times 1.3 \mathrm{~mm})$ were made through the structure [see Fig. 2(c)]. The grating was magnetized by two SmCo magnets to generate a magnetic field in the slots. The resolution of the resonance induced by $N$ cycles of a periodic field is estimated by the Fourier transform of the field and is $\sim 1 / N$ (FWHM) [29]. Therefore, it is $10 \%$ for $N=10$.

The magnetic field calculations in Figs. 3(a) and 3(b) show that the amplitude reaches $B_{a}>0.12 \mathrm{~T}$ for $B_{z}$ at $z \geq 0 \mu \mathrm{m}$, while $B_{a}>0.1 \mathrm{~T}$ for $B_{x}$ at $z \geq 9 \mu \mathrm{m}$. These amplitudes are large enough to induce the transition in $o$-Ps within 50 ps. The amplitude $B_{a}=0.12 \mathrm{~T}$ corresponds to the magnetic energy flux of a continuous electromagnetic wave, $c B_{a}^{2} / 2 \mu_{0} \sim 170 \mathrm{MW} \mathrm{cm}^{-2}$, where $c$ is the speed of light and $\mu_{0}$ is the vacuum magnetic permeability. For comparison, this flux is much larger than that accumulated in a Fabry-Perot cavity by a strong milliwave source, reported to be around $10 \mathrm{~kW} \mathrm{~cm}^{-2}$ [30]. The magnetic grating works as a passive component without any power supply unlike lasers and microwaves.

A biased field $B_{0} \sim 1.0 \mathrm{~T}$ of $B_{z}$ in Fig. 3(a) causes a Zeeman shift [33]. The energy eigenvalues of $|1,0\rangle$ and $|0,0\rangle$ are shifted depending on $B_{0}$, as shown in Fig. 3(c), where the first and second numbers in the ket vector express $S$ and its substate, respectively. In $B_{0}$, their eigenstates are expressed instead by $|+\rangle$ and $|-\rangle$. In our experiment, two transitions at around 207 and $211 \mathrm{GHz}$ are 


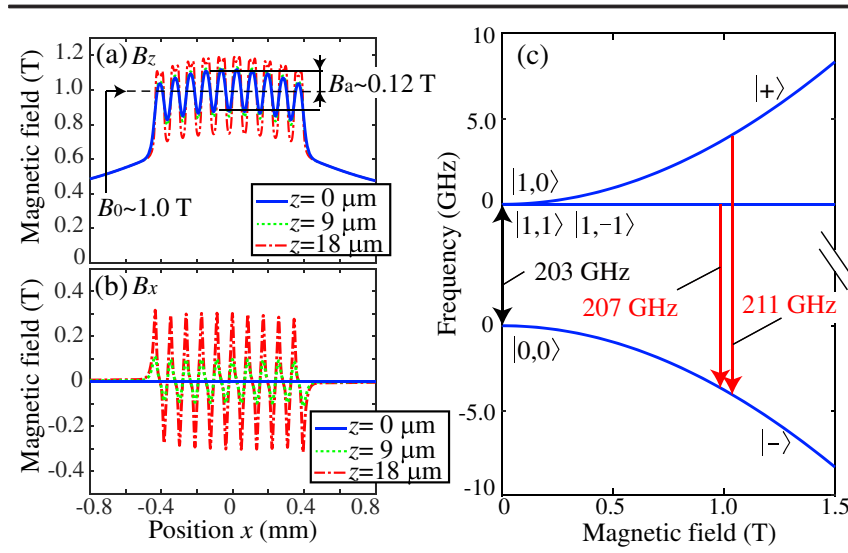

FIG. 3. The magnetic field distribution of the grating and Zeeman diagram of Ps. (a) Calculated magnetic fields $B_{z}$. The different lines show the $z$ position dependence of 0,9 , and $18 \mu \mathrm{m}$ from the slot center. The calculation was performed by using a 3D magnetostatics computation software [31,32]. (b) Same as (a) but for $B_{x}$. (c) Zeeman diagram of the 4 spin states of Ps atoms, which split into 3 energy levels, depending on $B$.

expected. It is to be noted that the loss of Ps signals off resonance due to the singlet-triplet mixing is negligible because the path length in $B_{0} \sim 1.0 \mathrm{~T}$ is only about $1 \mathrm{~mm}$ and the fraction of the $|+\rangle$ state Ps which self-annihilates there is negligible.

The resolution of the resonance is increased due to the nonuniformity of $B_{0}$ and $B_{a}$. A difference in $B_{0}$ changes the resonance frequency, following from the Zeeman effect. In the case of $z=0$ in Fig. 3(a), the frequency changes by about $1 \mathrm{GHz}$ for a shift in $B_{0}$ from 0.9 to $1.0 \mathrm{~T}$ during the passage of the Ps atoms. What frequency induces the most efficient transition is estimated from the Rabi oscillation, which depends on $B_{a}$. This effect is around $2 \%$ of the resonance frequency and is negligible compared to its $10 \%$ resolution.

The experiment was performed by using a high-quality energy-tunable Ps beam [23], based on the photodetachment of accelerated Ps negative ions $\left(\mathrm{Ps}^{-}\right)$[34-36] generated from pulsed slow positrons [37], as shown in Fig. 4. The $o$-Ps counts were monitored when $o$-Ps was either passing or not through the grating alternately and repeatedly. The velocity spread of the Ps beam is a few percent, as estimated from the momentum recoil of the photodetachment of $\mathrm{Ps}^{-}$. This effect is typically a few percent [23] and is negligible with respect to the $10 \%$ resolution.

Figure 5(a) shows the Ps beam profile at $2.0 \mathrm{keV}$ without the grating, measured by a microchannel plate (MCP), after subtraction of the background $\gamma$ rays by applying a time-of-flight analysis [29]. The profile when the grating was moved onto the beam axis is shown in Fig. 5(b). Figures $5(\mathrm{c})$ and $5(\mathrm{~d})$ are the $y$ and $z$ projections of Fig. 5(b), respectively. The FWHMs of the $o$-Ps distributions along the $y$ and $z$ axes were 1.42 and $1.17 \mathrm{~mm}$, respectively, and were slightly larger than the sizes of the

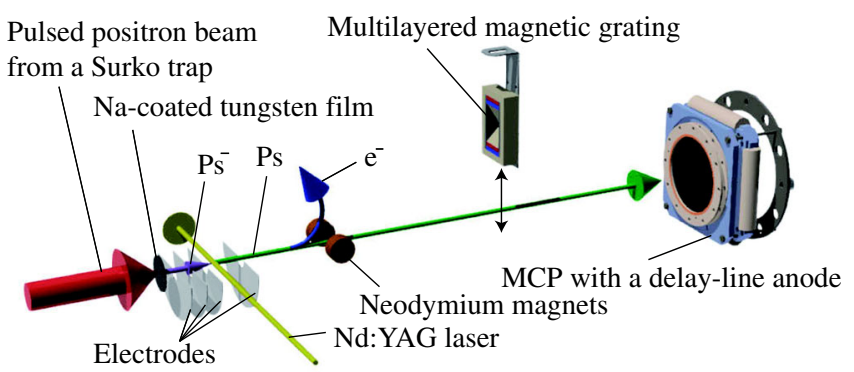

FIG. 4. The main part of our experimental setup, including the production of a high-quality energy-tunable Ps beam [23]. A pulsed positron beam from a Surko-type trap [37] with a frequency of $50 \mathrm{~Hz}$ is implanted into a tungsten film with a thickness of $100 \mathrm{~nm}$ and a submonolayer $\mathrm{Na}$ coating to produce Ps negative ions $\left(\mathrm{Ps}^{-}\right)$[34-36]. $\mathrm{Ps}^{-}$are photodetached by a $\mathrm{Nd}$ : YAG laser synchronized with the positron beam to produce Ps atoms. By changing the $\mathrm{Ps}^{-}$acceleration voltage applied on the electrodes, an energy-tunable monoenergetic Ps beam from 0.2 to $3.3 \mathrm{keV}$ is produced. These Ps atoms are detected by a 2D position sensitive detector, which consists of a microchannel plate (MCP) and a delay-line anode to measure the Ps beam profile. The electrons ejected by the photodetachment are separated by the magnetic field generated by two permanent magnets. The magnetic grating is mounted on a linear transfer. When the grating is moved onto the Ps beam axis, the Ps atoms pass through the slots of the grating and experience a time-dependent oscillating magnetic field.

slots, i.e., 1.30 and $1.05 \mathrm{~mm}$. To obtain the Ps counts $I$ having experienced the magnetic field, we defined a circle of radius $1.3 \mathrm{~mm}$ centered on the average position $P$, so that all Ps counts in Fig. 5(b) were included. The background was 2 orders of magnitude lower than the Ps counts [29].

The value $I$ was normalized by using the Ps counts in the absence of the periodic field $I_{0}$ in Fig. 5(a), because $I$ depended on $v$. Since $I_{0}$ had a position dependence, the counts at around $P, I_{0}^{P}$, needed to be considered. We defined an ellipse centered on $P$ characterized by a major axis of $2.1 \mathrm{~mm}$ and a minor axis $1.8 \mathrm{~mm}$ [full width tenth maximum of Figs. 5(c) and 5(d), respectively]. We calculated $I_{0}^{P}$ by summing the Ps counts inside this ellipse. Then the Ps counts corresponding to the area of the slots $A_{\text {slot }}$ were calculated as $I_{s}=I_{0}^{P} A_{\text {slot }} / A_{\text {ellipse }}$, where $A_{\text {slot }}=$ $0.61 \mathrm{~mm}^{2}$ was the designed value and $A_{\text {ellipse }}$ the area of the ellipse. The normalized Ps counts $I_{n}$ were obtained by $I / I_{s}$.

Figure 5(e) shows $I_{n}$ as a function of $f$. We clearly observed the resonance signal, indicated by the dip centered at around $211 \mathrm{GHz}$. The spectrum exceeded 1, despite $I_{n}$ being already normalized, and had a slope, which gradually increased as $f$ became higher.

To understand this spectrum shape, we constructed a fit model. We used a Gaussian function for the resonance shape. The slope of the spectrum was interpreted as the energy-dependent change of the beam intensity. Since pileup events of Ps atoms were not reconstructed by the 


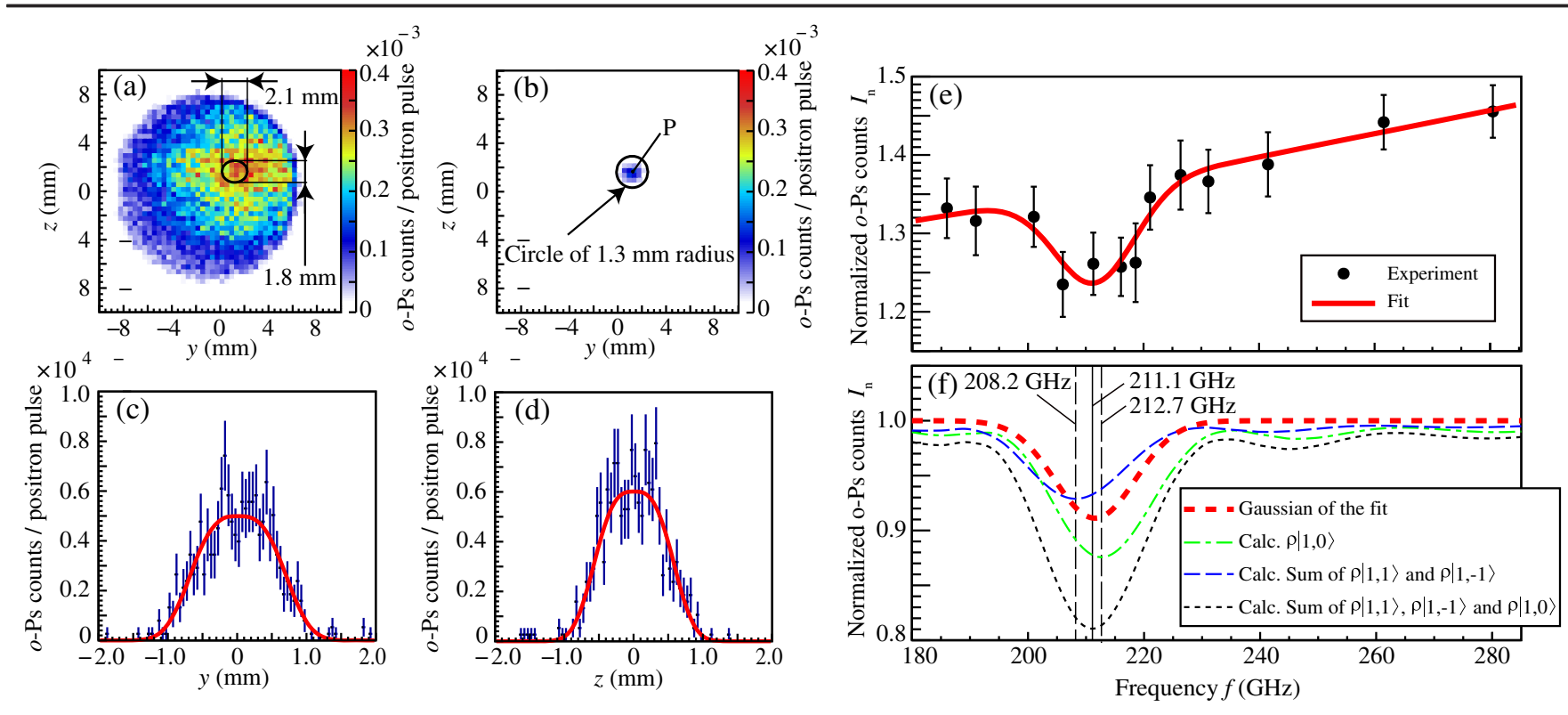

FIG. 5. Experimental results. (a) Beam profile of the Ps atoms at $2.0 \mathrm{keV}$, when the grating was moved away from the beam axis, measured by a microchannel plate.(b) Same as (a) but for the grating moved on the beam axis. (c) $y$ projection of (b). The fit function is $D \exp \left\{-\left|y-y_{0}\right|^{3} / 2 \sigma_{y}^{3}\right\}$, where $D, y_{0}, \sigma_{y}$ are fit parameters. FWHM $=1.4 \mathrm{~mm}$. The full width tenth maximum (FWTM) was $2.1 \mathrm{~mm}$. (d) $z$ projection of (b). FWHM $=1.2 \mathrm{~mm}$. FWTM $=1.8 \mathrm{~mm}$. (e) Normalized $o$-Ps counts $I_{n}$ as a function of $f$, which is derived from $f=\gamma v / a$. The black circles and the red line show the experimental results and the fit, respectively. (f) Comparison between experimental results and calculations. The red dashed line shows the Gaussian component of the fit of the experimental results. The green dot-dashed and blue dashed lines express the final population $\rho$ calculated for $|1,0\rangle$ and that for the sum of $|1,1\rangle$ and $|1,-1\rangle$, respectively. Their resonance frequencies are shown by the two dashed vertical lines at 208.2 and $212.7 \mathrm{GHz}$. The black short dashed line shows $\rho$ calculated for the sum of $|1,1\rangle,|1,-1\rangle$, and $|1,0\rangle$. The resonance frequency is $211.1 \mathrm{GHz}$, as shown by the solid vertical line.

delay-line anode, they were removed in the analysis. While $I$ is not affected by the pile up of Ps counts, $I_{0}$ is affected because the Ps counts without the grating are much higher. If we assume that $R$ is the average number of Ps atoms which produce MCP signals in one pulse of the positron beam, $I_{0} \propto R(1-R)$ and, hence, $I_{n} \propto 1 /(1-R) . R$ is proportional to the number of $\mathrm{Ps}^{-}$, i.e., $\propto \exp (-t / \gamma \tau)$, where $t$ is the time interval between the acceleration and the photodetachment and $\tau$ the lifetime of $\mathrm{Ps}^{-}(479 \mathrm{ps})$. The time $t$ is expressed as $t=L / v=L \gamma / f a$, with $v=f a / \gamma$, where $L$ is the effective length $(18 \mathrm{~mm})$, determined by the trajectory calculations of $\mathrm{Ps}^{-}$through the electrodes. With respect to the spectrum exceeding 1 , we define a proportionality factor $\epsilon$. From these considerations, the fit model is expressed as $\epsilon\left[A \exp \left(\left(f-f_{0}\right)^{2} / 2 \sigma^{2}\right)+1\right] /[1-C \exp (-L / f a \tau)]$, with fitting parameters $f_{0}, \sigma, A, C$, and $\epsilon$.

The fit result in Fig. 5(e) shows that the resonance frequency is $f_{0}=211.5 \pm 1.9 \mathrm{GHz}$, while the systematic uncertainty caused by $a$ is $\pm 1.0 \mathrm{GHz}$. The width of the resonance of $16 \pm 5 \mathrm{GHz}$ (FWHM) is comparable to the expected resolution of $21 \mathrm{GHz}$, calculated from $f_{0} / N$. Then, $\epsilon=1.23 \pm 0.05$, which is partly explained by the $10 \%$ or so increase in the beam size in both $y$ and $z$ directions, calculated as 1.2. Finally, $C=0.7 \pm 0.2$, which is comparable to the estimate of the typical Ps production rate of $0.1-0.15 /$ pulse, calculated as $0.8-1.2$.
To evaluate the resonance frequency in more detail, we solved the quantum Liouville equation including the Ps annihilation [29]. We assumed an initial population of three spin states of $o$-Ps atoms that were equally distributed at $\boldsymbol{B}=\mathbf{0}$. The two dashed vertical lines in Fig. 5(f) express the resonance frequencies of two candidate transitions, calculated as 208.2 and $212.7 \mathrm{GHz}$. The solid vertical line expresses the resonance frequency of the sum of three $o$-Ps populations, calculated as $211.1 \mathrm{GHz}$. The experimental result $f_{0}$ (see red dashed line) agreed with this calculation value within the uncertainty. It is noted that the measured dip depth was half that of the calculation (see black short dashed line). This may indicate that, in turn, the actual magnetic energy flux generated by the grating is half that of the calculation, considering the $B_{a}^{2}$ dependence of the Rabi oscillation. For $B_{a}=0.12 \mathrm{~T}$ at $z=0$, the flux is $85 \mathrm{MWcm}^{-2}$. This reduction may be explained by the characteristics of the cut surface of the grating slots, which were obtained by partly melting or vaporizing the grating by electrical discharge machining. Such a surface layer is made of a mixture of carbon steel and copper, which can smear and decrease $B_{a}$, while $B_{0}$ would be unchanged.

In conclusion, we have shown the observation of the motion-induced transition of the Ps atoms in the sub- $\mathrm{THz}$ region by using an energy-tunable Ps beam in combination with a multilayered magnetic grating. The microfabricated 
grating had through slots and a microsized periodic structure made of ferromagnetic material. The presence of the slots enabled the beam to maintain its coherence without collisions. This made it feasible to apply the motion-induced transition even to the exotic and the purely leptonic atom Ps, that contains an antiparticle. The microstructure generated a microsized periodic magnetic field where Ps experienced an oscillating field in the sub- $\mathrm{THz}$ region. Its amplitude corresponded to a magnetic energy flux of a continuous electromagnetic wave as high as $\sim 100 \mathrm{MW} \mathrm{cm}^{-2}$, resulting in the efficient magnetic dipole transition (electric dipole forbidden) in the sub-THz region. This component had the advantage of working as a highpower passive component without any power supply unlike lasers and microwaves. We expect that in the future this powerful method might also be applied to other atoms, ions, and molecules in various frequency regions.

It should be noted that the resonance width can be narrowed further by stacking more layers [20]. When the width is narrower than the difference between 2 transition frequencies described in this study, the $o$-Ps Zeeman substates can selectively transition to $p$-Ps, depending on $f$. This means that the information of the polarization of the Zeeman substates of the $o$-Ps atoms can be obtained, and that $o$-Ps beams polarized in their substates may be produced. Additionally, although the bias field generated by the grating caused the Zeeman shift, this field can be reduced to zero by assembling the magnets alternately [20] with the aid of, for example, microassembly developed for photonic crystals [38]. This might enable us to study the motion-induced transition in a zero-bias field even in the sub-THz region.

This work was supported by JSPS KAKENHI Grants No. JP17H01074 and No. JP19K12645.

*yugo.nagata@rs.tus.ac.jp

[1] V. V. Okorokov, The coherent excitation of nuclei moving through a crystal, Yad. Fiz. 2, 1009 (1965); Sov. J. Nucl. Phys. 2, 719 (1966).

[2] V. V. Okorokov, Coherent excitation of optical spectra of atoms passing through a crystal, Zh. Eksp. Teor. Fiz., Pis'ma Red. 2, 175 (1965) [JETP Lett. 2, 111 (1965)], http://www .jetpletters.ac.ru/ps/1598/article_24492.shtml.

[3] S. Datz, C. D. Moak, O. H. Crawford, H. F. Krause, P. F. Dittner, J. G. del Campo, J. A. Biggerstaff, P. D. Miller, P. Hvelplund, and H. Knudsen, Resonant Coherent Excitation of Channeled Ions, Phys. Rev. Lett. 40, 843 (1978).

[4] D. S. Gemmell, Channeling and related effects in the motion of charged particles through crystals, Rev. Mod. Phys. 46, 129 (1974).

[5] F. J. G. de Abajo and P. M. Echenique, Resonant-Coherent Excitation of Channeled Ions, Phys. Rev. Lett. 76, 1856 (1996).

[6] T. Azuma, T. Ito, K. Komaki, Y. Yamazaki, M. Sano, M. Torikoshi, A. Kitagawa, E. Takada, and T. Murakami,
Impact Parameter Dependent Resonant Coherent Excitation of Relativistic Heavy Ions Planar Channeled in Crystals, Phys. Rev. Lett. 83, 528 (1999).

[7] C. Kondo, S. Masugi, Y. Nakano, A. Hatakeyama, T. Azuma, K. Komaki, Y. Yamazaki, T. Murakami, and E. Takada, Three-Dimensional Resonant Coherent Excitation of Nonchanneling Ions in a Crystal, Phys. Rev. Lett. 97, 135503 (2006).

[8] T. Azuma, Y. Takabayashi, C. Kondo, T. Muranaka, K. Komaki, Y. Yamazaki, E. Takada, and T. Murakami, Anisotropic X-Ray Emission from Heliumlike $\mathrm{Fe}^{24+}$ Ions Aligned by Resonant Coherent Excitation with a Periodic Crystal Potential, Phys. Rev. Lett. 97, 145502 (2006).

[9] Y. Nakai, Y. Nakano, T. Azuma, A. Hatakeyama, C. Kondo, K. Komaki, Y. Yamazaki, E. Takada, and T. Murakami, Dressed Atoms in Flight through a Periodic Crystal Field: X-VUV Double Resonance, Phys. Rev. Lett. 101, 113201 (2008).

[10] Y. Nakano, C. Kondo, A. Hatakeyama, Y. Nakai, T. Azuma, K. Komaki, Y. Yamazaki, E. Takada, and T. Murakami, Polarization Control in Three-Dimensional Resonant Coherent Excitation, Phys. Rev. Lett. 102, 085502 (2009).

[11] K. Kimura, H. Ida, M. Fritz, and M. Mannami, Resonant Coherent Excitation of Surface Channeled Ions, Phys. Rev. Lett. 76, 3850 (1996).

[12] N. Hatke, M. Dirska, M. Grether, E. Luderer, A. Robin, A. Närmann, and W. Heiland, Surface Channeling Experiments at $20 \mathrm{MeV}$ and Resonant Coherent Excitation of $\mathrm{N}^{6+}$ Ions, Phys. Rev. Lett. 79, 3395 (1997).

[13] C. Auth, A. Mertens, H. Winter, A. G. Borisov, and F. J. G. de Abajo, Resonant Coherent Excitation of Fast Hydrogen Atoms in Front of a LiF(001) Surface, Phys. Rev. Lett. 79, 4477 (1997).

[14] Y. Nakano, Y. Takano, T. Ikeda, Y. Kanai, S. Suda, T. Azuma, H. Brauning, A. Brauning-Demian, D. Dauvergne, Th. Stohlker, and Y. Yamazaki, Resonant coherent excitation of the lithiumlike uranium ion: A scheme for heavy-ion spectroscopy, Phys. Rev. A 87, 060501 (2013).

[15] T. Hadeishi, W. S. Bickel, J. D. Garcia, and H. G. Berry, Observations of Lamb Shifts in H Using Spatially Periodic Potentials, Phys. Rev. Lett. 23, 65 (1969).

[16] A. Hatakeyama, Y. Enomoto, K. Komaki, and Y. Yamazaki, Motion-Induced Magnetic Resonance of $\mathrm{Rb}$ Atoms in a Periodic Magnetostatic Field, Phys. Rev. Lett. 95, 253003 (2005).

[17] Y. Kobayashi and A. Hatakeyama, Magnetic resonance of atoms passing through a magnetic lattice, J. Phys. Conf. Ser. 185, 012021 (2009).

[18] Y. Kobayashi, Y. Shiraishi, and A. Hatakeyama, Spin nutation induced by atomic motion in a magnetic lattice, Phys. Rev. A 82, 063401 (2010).

[19] A. Hatakeyama and K. Goto, Atomic spin resonance in a rubidium beam obliquely incident to a transmission magnetic grating, J. Phys. B 49, 065005 (2016).

[20] Y. Nagata, S. Kurokawa, and A. Hatakeyama, Magnetic resonance of rubidium atoms passing through a multilayered transmission magnetic grating, J. Phys. B 50, 105002 (2017).

[21] M. M. Agamalyan, G. M. Drabkin, and V. I. Sbitnev, Spatial spin resonance of polarized neutrons. A tunable slow neutron filter, Phys. Rep. 168, 265 (1988). 
[22] E. Jericha, J. Bosina, P. Geltenbort, M. Hino, W. Mach, T. Oda, and G. Badurek, Neutron detection in the frame of spatial magnetic spin resonance, Nucl. Instrum. Methods Phys. Res., Sect. A 845, 552 (2017).

[23] K. Michishio, L. Chiari, F. Tanaka, N. Oshima, and Y. Nagashima, A high-quality and energy-tunable positronium beam system employing a trap-based positron beam, Rev. Sci. Instrum. 90, 023305 (2019).

[24] T. Yamazaki, A. Miyazaki, T. Suehara, T. Namba, S. Asai, T. Kobayashi, H. Saito, I. Ogawa, T. Idehara, and S. Sabchevski, Direct Observation of the Hyperfine Transition of Ground-State Positronium, Phys. Rev. Lett. 108, 253401 (2012).

[25] A. Miyazaki, T. Yamazaki, T. Suehara, T. Namba, S. Asai, T. Kobayashi, H. Saito, Y. Tatematsu, I. Ogawa, and T. Idehara, First millimeter-wave spectroscopy of ground-state positronium, Prog. Theor. Exp. Phys. (2015) $011 \mathrm{C} 01$.

[26] A. P. Mills, Jr. and G. H. Bearman, New Measurement of the Positronium Hyperfine Interval, Phys. Rev. Lett. 34, 246 (1975).

[27] D. B. Cassidy, T. H. Hisakado, H. W. K. Tom, and A. P. Mills, Jr., Positronium Hyperfine Interval Measured via Saturated Absorption Spectroscopy, Phys. Rev. Lett. 109, 073401 (2012).

[28] A. Ishida, T. Namba, S. Asai, T. Kobayashi, H. Saito, M. Yoshida, K.Tanaka, and A.Yamamoto, New precision measurement of hyperfine splitting of positronium, Phys. Lett. B 734, 338 (2014).

[29] See Supplemental Material at http://link.aps.org/ supplemental/10.1103/PhysRevLett.124.173202 for the
Rabi oscillation, the resolution of the resonance line, a time-of-flight analysis, and the quantum Liouville equation.

[30] A. Miyazaki, Direct measurement of the hyperfine structure interval of positronium using high power millimeter wave technology, Ph.D. thesis, The University of Tokyo, 2013.

[31] P. Elleaume, O. Chubar, and J. Chavanne, Computing 3D magnetic field from insertion devices, Proc. Conf. 3, 3509 (1997).

[32] O. Chubar, P. Elleaume, and J. Chavanne, A 3D magnetostatics computer code for insertion devices, J. Synchrotron Radiat. 5, 481 (1998).

[33] M. Charlton and J.W. Humberston, Positron Physics (Cambridge University Press, Cambridge, England, 2009).

[34] Y. Nagashima, Experiments on positronium negative ions, Phys. Rep. 545, 95 (2014).

[35] Y. Nagashima, T. Hakodate, A. Miyamoto, and K. Michishio, Efficient emission of positronium negative ions from Cs deposited W(100) surfaces, New J. Phys. 10, 123029 (2008)

[36] H. Terabe, K. Michishio, T. Tachibana, and Y. Nagashima, Durable emission of positronium negative ions from Na- and K-coated W(100) surfaces, New J. Phys. 14, 015003 (2012).

[37] C. M. Surko and R. G. Greaves, Emerging science and technology of antimatter plasmas and trap-based beams, Phys. Plasmas 11, 2333 (2004).

[38] S. Noda, K. Tomoda, N. Yamamoto, and A. Chutinan, Full three-dimensional photonic bandgap crystals at near-infrared wavelengths, Science 289, 604 (2000). 